\title{
De la génération de l'idée au lancement du produit : comment les stratégies de propriété intellectuelle et l'environnement professionnel peuvent doper
} l'innovation

\author{
From ideation to product launch: how IP strategies and work \\ environment can foster innovation
}

\author{
Maggy Perrier ${ }^{1}$, Audrey Depeige ${ }^{2}$ \\ ${ }^{1}$ Essilor, Paris, France, perrierm@essilor.fr \\ 2 Télécom Ecole de Management, Evry, France, depeig_a@telecom-em.eu
}

RÉSUMÉ. Ce manuscrit présente les processus et méthodes par lesquelles une société internationale, le groupe Essilor, crée et capture de la valeur par ses innovations. L'article décrit les activités et la structure de l'entreprise, contribuant à l'amélioration des performances en matière d'innovation, et au maintien de son avantage compétitif. Le contexte concurrentiel pousse notamment l'entreprise à rechercher diverses formes de collaborations avec d'autres organisations afin de créer, capturer, et offrir de la valeur (produits ou services) aux porteurs de verres de lunettes, en encourageant l'innovation fondée sur la connaissance. En particulier, les illustrations terrain montrent que l'entreprise met à profit deux sources d'accès aux nouvelles technologies, recourant à la mise en réseau avec d'autres entreprises de son écosystème susceptibles de stimuler le co-développement de nouveaux produits. De nouvelles formes de partenariats se traduisent ici par l'émergence d'un apprentissage inter-organisationnel et la diffusion des nouvelles connaissances, appuyées par des stratégies IP dédiées. ABSTRACT. This manuscript presents the processes and methods by which an international company, Essilor creates and captures value through its innovations. The article describes the activities and structure of the company, contributing to the improvement of innovation performance, and maintaining its competitive advantage. The competitive environment urges the company to seek various forms of collaborations with other organizations to create, capture, and deliver value (products or services) to spectacle lens wearers, encouraging knowledge-based innovation. In particular, field illustrations show that the company utilizes two sources of access to new technologies, using the networking with other companies in its ecosystem could stimulate the co-development of new products. New forms of partnerships are illustrated by the emergence of an inter-organizational learning and the dissemination of new knowledge, supported by dedicated IP strategies.

MOTS-CLÉS. Innovation, connaissance, processus, propriété intellectuelle, écosystème, collaboratif, ressources.

KEYWORDS. Innovation, knowledge, processes, intellectual property, ecosystem, collaborative, resources.

L’innovation est régie par la capacité à identifier des liens, à découvrir des opportunités et à en tirer profit, en ouvrant de nouveaux débouchés (dans lesquels la technologie joue souvent un rôle essentiel) ou en inventant des méthodes inédites aptes à satisfaire les besoins de marchés déjà existants. Un exemple intéressant de ce type de perfectionnement est donné, pour mémoire, dans le contexte de l'innovation frugale à destination des pays en développement, par une paire de lunettes munie de verres réglables par le porteur afin de satisfaire son besoin spécifique (TIDD, BESSANT, \& PAVITT, 2006), sans nécessiter de recourir à un opticien dont la pénurie peut être aiguë (la technique de fabrication est conçue pour maximaliser le rapport qualité/prix). La suite de l'article est consacrée à un environnement d'entreprises à haute technologie dans le même domaine que l'exemple précédent, mais dans l'axe de la recherche de l'excellence technologique. L'industrie ophtalmique est caractérisée par une complexification croissante de ses produits de correction visuelle portée par des connaissances de plus en plus pointues impliquant toutes les phases de la conception à la fabrication. Cette tendance lourde amène des questionnements sur les stratégies d'internalisation/externalisation des activités intenses en connaissances, d'autant plus aigus que le domaine est très concurrentiel. La problématique de ces stratégies va donc s'articuler autour de l'axe de collaboration externe dans le management de l'innovation, axe dans lequel la contractualisation de droits de propriété intellectuelle va se révéler essentielle, tout autant que la stimulation des collaborateurs 
(à l'intérieur de la société) par l'apport de connaissances externes. En effet, concernant cet apport, des travaux récents ont montré que l'accès à des connaissances géographiquement localisées influencait directement les capacités d'innovation des entreprises, les niveaux d'investissements de R\&D aussi bien que la quantité et la nature des connaissances acquises à l'extérieur, en relation avec la valeur du capital social (LAURSEN, MASCARIELI et PRENCIPE 2012b). L'objet de l'article est ainsi d'analyser cette problématique en l'illustrant par la mise en perspective du cas de la société Essilor.

A cet égard, il apparaît primordial pour l'organisation d'expliquer à la fois les opportunités et les défis auxquels sont confrontés les ingénieurs quand ils s'efforcent de collecter et de combiner des connaissances (MURRAY et O'MAHONY 2007). De plus, l'entreprise va probablement bénéficier de l'inclusion de facteurs socio-écologiques dans ses efforts à comprendre et à évaluer systématiquement son contexte concurrentiel. De tels efforts dopent la performance d'innovation via la génération et l'implantation d'une stratégie d'anticipation (PETRINI et POZZEBON 2009), mais ceci soulève la question de l'appropriation de cet apport et de son intégration par les ingénieurs dans la procédure d'innovation de leur entreprise : le processus d'imbrication de ces différents blocs de connaissances à l'intérieur d'une innovation réussie se déroule dans des conditions de forte incertitude et gérer l'innovation va consister à transformer ces incertitudes en nouvelles connaissances (TIDD, BESSANT, \& PAVITT, 2006).

Cette analyse va être menée en trois étapes toutes illustrées par l'exemple de la société Essilor. La première étape pose le cadre de l'innovation collaborative, en partant des mécanismes d'innovation activés par les acteurs internes puis en montrant leur enrichissement par l'apport extérieur. La deuxième partie aborde les enjeux organisationnels en approfondissant leurs aspects internes à l'entreprise. La gestion des droits de propriété intellectuelle, tant pour l'activité interne que pour l'activité collaborative externe fait l'objet de la troisième partie. La conclusion met en valeur les défis de la gestion de l'innovation dans une entreprise internationale et les solutions portées par la stratégie de propriété intellectuelle considérée comme le bras armé de la stratégie d'innovation collaborative régissant les échanges de connaissances et permettant leur transformation en valeur. Elle met ainsi au jour que la réussite de l'innovation collaborative est fortement dépendante de l'organisation et de la qualité de l'apprentissage opéré.

\section{La recherche d'un renouvellement permanent : l'innovation collaborative au cœur de la stratégie d'entreprise}

La pression de compétition aiguë a poussé les organisations à adopter des systèmes dopant le transfert et la rétention de connaissances ainsi qu'à créer de la valeur par la création de connaissances. Dans une perspective théorique de l'innovation fondée sur la connaissance, l'innovation est définie comme un résultat duplicable, nouveau et utile issu des connaissances (QUINTANE, et al. 2011). Le déploiement, l'intégration et l'utilisation des ressources en connaissances intercèdent entre les actifs intangibles intraorganisationnels et les résultats en termes de croissance et performance de l'innovation (MAURER, BARTSCH et EBERS 2011). Tandis que de tels systèmes ont occasionné un regain d'attention de la part des chercheurs et des professionnels, les travaux de recherche menés par (VASUDEVA, SPENCER et TEEGEN 2013) suggèrent que les capacités concurrentielles et innovatives d'une entreprise peuvent être améliorées par des partenariats avec d'autres acteurs solides et possédant un capital social significatif. Ce faisant, les entreprise peuvent engendrer de la valeur et de la croissance aussi bien que des nouvelles connaissances technologiques et de la légitimité en gagnant l'accès à de nouvelles ressources de connaissances. Cependant, des travaux de recherche antérieurs montrent qu'une recherche aléatoire de ressources dans l'environnement de business peut ne pas apporter de résultats probants (BUTEL et WATKINS 2006). En effet, le panorama concurrentiel global impose aux entreprises de s'adapter au changement afin de tirer bénéfice des opportunités émergentes du marché global. A cet égard, il devient particulièrement critique d'établir de nouvelles collaborations et de nouveaux partenariats.

KETCHEN et al. ont avancé que l'innovation collaborative fournit aux grandes organisations des possibilités de relever de nouveaux défis, en exploitant des ressources telles que des réseaux, des capacités apprenantes ou en saisissant des opportunités, afin de partager, créer et développer des potentialités de (c) 2016 ISTE OpenScience - Published by ISTE Ltd. London, UK - openscience.fr 
connaissances et d'innovation (KETCHEN, IRELAND et SNOW 2007). Dans cette perspective, il apparaît que les organisations gagneraient à saisir des opportunités (issues en particulier d'évolutions technologiques) pour développer de nouveaux partenariats, qui les aideraient à s'adapter aux changements d'environnement. Spécifiquement, l'expérience du terrain a montré qu'un rythme rapide d'évolution des technologies et des marchés n'est pas (ou n'est plus) toujours suffisant pour assurer le développement de produits compétitifs par les seules ressources internes ${ }^{1}$. Comme le suggère un accroissement récent de l'initialisation de collaborations externes, les entreprises s'efforcent d'explorer des combinaisons d'expertise et de flexibilité en collaborant avec d'autres entreprises dans des réseaux d'innovation (GURAU, 2009). De tels scénarios émergent principalement de l'augmentation de la complexité des procédures d'innovation et des technologies, qui ne sont plus nécessairement accessibles en interne. Pour illustrer ce point de vue, Essilor tire parti de partenariats de longue date (ou plus récents) avec des universités locales, qui permet à ses innovateurs internes de davantage développer leurs connaissances, leurs capacités et leurs réseaux, en optimisant en même temps les investissements et les coûts opérationnels du développement de produit. Le développement du produit Prevencia ${ }^{\circledR}$ illustre bien ce point, pour lequel Essilor a mené une collaboration en amont avec l'Institut de la vision, devenant ainsi le premier fabricant de verres à lancer sur le marché un verre non teinté à protection oculaire par filtrage des UV et du rayonnement bleu des écrans avec une faible réflexion face arrière. De surcroît, afin d'obtenir la maturation de l'idée en produit d'innovation, la société suit une procédure détaillée avec des étapes pré-définies telles la collecte et l'identification des connaissances pertinentes à acquérir de l'environnement extérieur, qui seront ré-utilisées à la résolution de défis internes de business aussi bien qu'à contribuer à l'amélioration de ses performances actuelles. Une procédure de découverte et de formalisation d'idées, ayant vocation à transformer les idées en inventions applicables, a ainsi été déployée (Cf. Figure 1 ci-dessous).

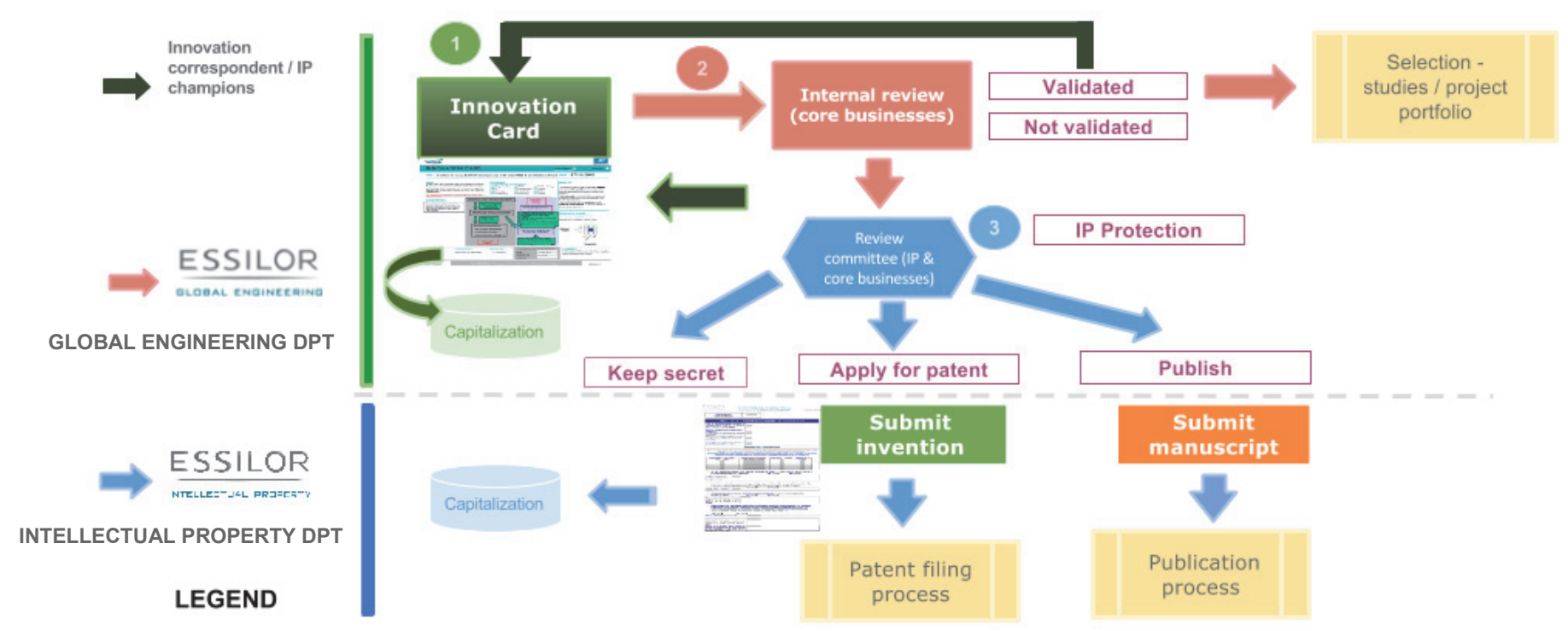

Figure 1. Une illustration de la procédure d'innovation mise en place chez Essilor - (Source : société Essilor)

En premier lieu, il est clair que la transformation d'une idée créative en innovation acceptable par le marché requiert un important travail pour que le produit final devienne un best-seller. Pour faciliter le travail de description et d'identification des nouvelles idées très tôt dans le déroulement de la procédure, Essilor a développé une « fiche-innovation » qui décrit brièvement et illustre graphiquement l'idée. La vocation de la fiche-innovation est de capturer «l'idée inventive » qui deviendra un produit ou un outil de conception en s'adossant à la vision de l'innovation définie par Essilor. Ainsi, l'auteur de l'invention est encouragé à faire la présentation de son idée sous forme de fiche-innovation et, pour

1. La dynamique des ressources internes, en termes de mise en œuvre de stratégie de connaissances en mode tant d'exploration que d'exploitation, peine à suivre la dynamique des marchés et des technologies de pointe. 
cela, il est constamment guidé par des correspondants Innovation et champions Propriété Intellectuelle (1). Cette phase illustre bien l'autonomie professionnelle, en ce sens que chaque inventeur est encouragé à faire émerger des idées tout au long de la procédure d'innovation. Pendant que la phase de génération d'idée se focalise sur les méthodes dynamiques et inter-équipe de génération collective d'idées par sessions de brainstorming, les étapes suivantes seront dédiées à l'évaluation des idées, à leur intégration et à la construction d'innovation par le biais de fiche-innovation (2). Durant ces phases, une analyse par type business est menée en profondeur et les opportunités de génération de droits de propriété intellectuelle sont identifiées. Comme les ingénieurs raisonnent par analogies et s'arrêtent à des solutions très tôt dans la phase de conception, des méthodes spécifiques de résolution inventive de problèmes sont mises en œuvre en complément des méthodes de génération d'idées pour dériver des idées créatives des solutions existantes (typiquement, les ingénieurs se reposent sur des méthodes de Design thinking ou des outils « Concept-Knowledge driven »). Il est alors demandé à l'équipe d'ingénieurs en charge du projet d'analyser la faisabilité de l'idée et de brosser des possibilités de fabrication et de distribution du produit. Les idées ayant réussi à passer avec succès cette analyse sont amenées à suivre la procédure IP, selon trois directions possibles (3), procédure qui est transverse aux différents départements et qui est supportée par le Comité Exécutif.

Parallèlement aux procédures existantes, les managers sont évalués sur leur capacité tant à accompagner les collaborateurs dans leur génération de nouvelles connaissances qu'à exploiter des connaissances déjà disponibles à l'intérieur de l'entreprise (NICKERSON et ZENGER 2004). Ce postulat est aussi supporté par d'autres travaux, qui mettent en lumière que, même si la capacité d'une organisation à acquérir et à stocker des connaissances est absolument primordiale, elle ne conduit pas nécessairement à des résultats innovants, à moins que les connaissances n'aient été exploitées, traitées et re-combinées de telle sorte à préfigurer le développement d'idées innovantes et à accroître la probabilité pour des inventions d'atteindre un stade de maturité (JOSHI, et al. 2010). Dans de tels cas, les organisations, pour Provost et Fawcett, devraient diviser leurs problèmes de business en éléments plus petits pour pouvoir estimer les prospects et la valeur attendue de l'innovation (PROVOST et FAWCETT 2013). Pourtant, l'histoire de l'entreprise montre que les conditions pour une innovation cumulative s'établissent à des niveaux multiples (MURRAY et O'MAHONY 2007) : le niveau institutionnel (institutions de régulation), le niveau du terrain (le marché), le niveau organisationnel (l'entreprise) et le niveau de la communauté (la société). Au niveau institutionnel, Essilor collabore par exemple avec l'Université de Shanghai pour mettre en commun des compétences et moyens disponibles dans l'investigation et dans la conception de solutions dédiées. Au niveau du terrain, on peut citer le programme d'innovation sociale EyeMitra, qui consiste en un business model innovant déployé dans l'Inde rurale. Les initiatives et moyens mis en place au niveau organisationnel sont variées : les canaux de veille, les départements de recherche, les communautés techniques transverses, les laboratoires internes d'innovation et également le centre de développement conjoint (Nikon-Essilor International joint research center), grâce notamment à l'investissement significatif d'une partie du Chiffre d'affaires en R\&D pendant que d'autres structures internes, telles que le «business CSR (Corporate Social Responsibility)» sont venus renforcer les nombreux leviers à l'expertise technique et à la résolution collaborative de problèmes, pour développer des solutions de pointe concernant la vue. En se focalisant sur les innovations de business à impact social, Essilor a récemment lancé un challenge d'innovation ouverte nommé « See Change » visant à une production participative d'expertise parmi les universitaires, les scientifiques, les développeurs, les ingénieurs et le startups pour développer de nouvelles solutions à destination des 2.5 milliards de personnes n'ayant pas encore accès aux verres correcteurs.

Corrélativement, la recherche antérieure tend à indiquer que l'échange de capacités de connaissances et le développement des interactions interdisciplinaires entre les parties prenantes contribuent à la génération significative de résultats en termes à la fois d'innovation et de performance opérationnelle (HARGADON et SUTTON 1997), (OBSTFELD 2005). Il vient que cette approche stratégique peut doper l'innovation en de multiples points simultanément. En ce sens, la collaboration entre le monde des affaires, les agences gouvernementales, les institutions de recherche et les investisseurs représente 
un catalyseur pour échanger de nouvelles formes de connaissances, pour développer l'innovation top niveau, tout en créant de la valeur pour les co-créateurs d'innovation. A cet égard, les réseaux facilitent la circulation de flux d'informations au travers des frontières organisationnelles : des travaux de recherche mettent en lumière que les organisations en réseaux tendent à accroître la survivabilité des entreprises, tout en réduisant la compétition (SOLITANDER et TIDSTROM 2010). Ce point s'illustre chez Essilor par le croisement de réseaux de partenariats externes à l'entreprise (comme illustré plus haut), avec une politique d'acquisitions qui viennent enrichir des réseaux de distribution, de production et commerciaux. Cela permet au groupe d'offrir des produits et services innovants adaptés aux besoins des différents segments de consommateurs et des attentes des marchés locaux couvrant une très large part du business de la correction et protection de la vue. Des synergies entre les entités acquises et historiques sont systématiquement encouragées (dans les limites du respect du droit de la concurrence) pour nourrir l'innovation tout en optimisant les ressources nécessaires. Pour aller plus loin, il semble bien que les interactions entre des services différents permettent de constituer un terreau dans un service spécifique, qui viendra lui-même fertiliser les connaissances locales pour faire émerger de nouvelles connaissances. De tels mécanismes indiquent que la pertinence des connaissances détenues par certains services va probablement influer sur la quantité et la destination des connaissances diffusées. Ainsi, ALCATER et ZHAO (2010) défendent l'idée selon laquelle les liens étroits entre des sites industriels permet aux entreprises de décupler les connaissances pour des avantages compétitifs - en mutualisant « les avantages d'un site particulier, tels que la proximité avec des universités, les politiques gouvernementales, un capital humain abondant »-tandis que les innovations provenant d'entreprises co-localisés avec leurs concurrents tendent à être rapidement internalisées.

De plus, les résultats de la recherche antérieure indiquent que l'évolution dans un environnement permettant l'accès à de nouvelles ressources et opportunités est un facteur-clé de l'innovation pour les entreprises (HEARN \& PACE, 2006), ce qui est consistant avec les résultats de (COOMS, DEEDS et DUANE IRELAND 2009) ou de (HMIELSKI et CORBETT 2008) qui ont soutenu que l'arrière-plan géographique de l'organisation décuple la performance dans des environnements dynamiques, du fait soit de stratégies de recherche localisées et internationales soit de recherches internes stables. De même, la recherche académique indique que le niveau d'expérience internationale d'une entreprise réduit défavorablement les résultats d'une étude sur l'extension de l'internationalisation, en expliquant que «moins une entreprise a de connaissances expérientielles, plus significatifs sont les effets des connaissances initiales et des connaissances acquises par le biais de partenaires-clés » (BRUNEEL, YLI-RENKO et CLARYSSE 2010). Pour compléter sur le point sur l'impact positif des réseaux, Essilor maintient en même temps un équilibre entre des services et fonctions locales, et des équipes «corporate » dont le périmètre d'action et l'implantation internationale contribuent largement à la capitalisation et la diffusion des connaissances, des savoir-faire et des bonnes pratiques au sein de l'entreprise.

Plus spécifiquement, plusieurs études soulignent l'influence bénéfique de l'environnement extérieur de l'entreprise ainsi que des capacités individuelles, sur l'efficience des deux types d'innovation (exploration et exploitation) et sur l'amélioration de l'apprentissage de leurs perspectives (KANE et ALAVI 2007). Toutefois, la performance de l'organisation dépend de l'établissement effectif d'un équilibre entre les deux types de dynamique que sont la stabilité organisationnelle et l'apprentissage. En plus de ses investissements en R\&D et de ses brevets, la stratégie d'innovation de l'entreprise peut inclure la création de valeur et l'accès à des technologies spécifiques par le biais de fusions/acquisitions, en se reposant ainsi sur les connaissances externes : pourtant, il est peu probable que " la stratégie d'innovation puisse être achetée sur la base d'une simple logique make or buy » (ARORA, BELENZON et RIOS 2013). L'exemple de l'entrée d'Essilor sur le marché des «Readers » (lunettes corrigeant la vision de près et de prescription fixée) illustre ce choix stratégique de développer cette activité acquise au travers du rachat de la société FGX en mettant au catalogue une sélection des innovations de ses fournisseurs. L'externalisation est donc un moyen rapide et optimisé d'offrir à ses clients les dernières innovations en complément à des activités de $R \& D$. 


\section{Un environnement interne soutenant l'innovation}

A la lumière des exemples précédents, il est clair qu'un aspect clé de l'innovation réside dans le succès des applications commerciales et de la soumission constante et systématique de nouvelles idées au marché (SHANE 2003). KURATKO, HORNSBY et COVIN (2013) identifient cinq aspects de l'environnement interne de l'entreprise : le support de la direction générale, l'autonomie dans le travail, les récompenses, la disponibilité de temps et les frontières organisationnelles. Ses travaux mettent en évidence le rôle d'outils de diagnostic pour évaluer les dimensions contribuant à créer un environnement pour les équipes propice à l'innovation (audits de maturité et d'innovation), qui permettent d'évaluer la maturité de l'entreprise relativement à ces cinq facteurs et de faire ressortir les zones de l'entreprise nécessitant des efforts soutenus de développement. Ces auteurs complètent cette vue en posant qu'il incombe à la direction générale de créer un environnement de travail « hautement favorable aux comportements d'entrepreneurs et d'innovateurs » (p. 39). Pour illustrer ce point, il semble bien que de nouvelles opportunités puissent émerger sous la forme de nouveaux produits, de nouveaux services, de nouveaux instruments / systèmes ou d'amélioration incrémentale de méthodes de production ou de produits existants. DRUCKER (1985) nota que la diversité, exprimée à travers un éventail de procédures complémentaires (procédures internes de créativité et d'innovation d'Essilor), de structures industrielles (partenariats d'Essilor avec des fournisseurs pour le développement d'innovations de moyens de fabrication par exemple), de démographies de marché (efforts d'intelligence compétitive), de changements dans les cadres de référence (déploiement de l'approche Design thinking) et de connaissances (déploiement de la méthode $\mathrm{C}-\mathrm{K}$ ) constituent la source de nouvelles opportunités conduisant à l'innovation. En rassemblant tout cela, nous voyons que les relations collaboratives fondées et développées sur ces valeurs peuvent mener au développement d'innovations confortant ou étendant le capital sous tous ses aspects (social, économique et environnemental) d'une entreprise, dont le personnel fournit la pierre angulaire. En particulier, KURATKO, HORNSBY et COVIN (2013) notent que les collaborateurs jouent un rôle clé en créant des environnements favorables à l'innovation, comme « des pratiques stimulantes réservant du temps à des approches sortant des sentiers battus et persévérant malgré les résistances organisationnelles (...) ». Ces auteurs insistent pour que les entreprises dédient de nombreux efforts à trouver des façons et des moyens pour soutenir un environnement de travail ouvert à l'innovation en dépit des changements incessants de l'environnement extérieur de l'entreprise.

- (1) Soutien de la direction générale : chez Essilor, un tel soutien organise les efforts constants pour encourager la culture de l'innovation, au travers de concours d'innovation ou de mesure des résultats d'innovation dans les « Balance Score Cards »;

- (2) Autonomie professionnelle : renforcer les liens inter-équipes de part et d'autre des frontières organisationnelles et faciliter la transformation des idées créatives en innovations aptes à être lancées sur le marché. Essilor a récemment introduit les « IP champions », dont le rôle consiste à assurer la promotion des solutions innovantes en faisant le pont entre les équipes opérationnelles (définissant la solution) et les équipes de Propriété Intellectuelle (évaluant la valeur technique de l'idée et sa brevetabilité) ;

- (3) Programmes de reconnaissance et rétribution des inventeurs : des récompenses matérielles (primes) et morales (cérémonie annuelle avec remise des trophées de l'innovation et des inventeurs) sont inscrites dans les programmes d'Essilor cherchant à encourager créativité et innovation dans l'entreprise ;

- (4) Disponibilité de temps : la gestion du portefeuille des projets chez Essilor prend en compte la maturité des solutions et un processus de sélection puis priorisation permet de dédier une partie des ressources sur des projets exploratoires et innovants ;

- (5) Frontières organisationnelles : pour compléter le dispositif des cartes innovations, un processus en entonnoir, combiné à une approche «stage gate », a été introduit pour maximiser les chances de collecter toutes les idées créatrices de valeur dans les différents départements du groupe, quel que soit l'auteur et d'être capable ensuite de donner les moyens et d'accompagner les ingénieurs dans le test de leurs idées de la preuve du concept au lancement sur le marché. Il s'agit de répartir les projets en fonction de leur maturité, puis de les faire avancer dans le processus (passages de " gates ») au regard de leur étape de développement, la sélection finale des projets se faisant sur la base de la valeur apportée à l'entreprise. Ce processus en entonnoir permet notamment d'optimiser les coûts, et dans le même temps, de garantir la réussite des projets sélectionnés. 
En ligne avec ces cinq dimensions, (SAINT-ONGE 1996) affirme que le croisement du capital humain, des ressources internes et des structures externes constitue la base de la création de valeur. Dit autrement, les entreprises qui réussissent sont celles qui sont capables de créer de la valeur en intégrant les connaissances tacites et les connaissances explicites, ainsi que l'expertise et la conscience des réalités extérieures. Une telle approche nécessite de transformer les connaissances existantes (implicites ou explicites) en des structures de connaissances plus grandes, qui élaborent des connaissances systémiques (NONAKA et TAKEUCHI 1995). SCHULZ (2003) note que la pertinence des nouvelles connaissances détermine jusqu'où celles-ci peuvent être connectées aux connaissances existantes. Selon ce dernier, les connaissances externes sont d'autant plus connectables aux connaissances locales que leur implication vers les connaissances locales est plus grande et plus facile à utiliser. A titre d'exemple, les équipes d'ingénierie sont amenées à explorer trois types différents de bases de connaissances, qui fournissent l'essentiel pour l'émergence des idées innovantes dans l'entreprise : les connaissances technologiques (les connaissances d'ingénierie et les aspects techniques des produits), les connaissances du marché (marketing et canaux de vente), connaissance de l'environnement concurrentiel (incluant les fournisseurs, les agences gouvernementales et les concurrents). Concrètement, les équipes d'ingénierie enrichissent leurs idées avec le soutien des fonctions transverses telles que «Techno Plans» et "Competitive Intelligence», incorporant le point de vue des fonctions amont telles que le Marketing, intégrant de ce fait les données d'analyses comparatives, d'intelligence économique et concurrentielle, les développements les plus récents du marché pour s'adapter aux besoins des porteurs de verres correcteurs. De plus, des « feuilles de route » (Road Maps) orientées consommateurs ont été créées au sein de la $R \& D$, visant à faire levier sur les connaissances acquises au niveau de l'organisation ainsi qu'à évaluer leur potentiel - fût-ce en termes de produits ou de développements de services - en ciblant les besoins des utilisateurs finaux. Les rôles dérivés de la feuille de route fonctionnent de façon transverse avec des équipes pluridisciplinaires. Autrement dit, les équipes techniques et scientifiques peuvent faire évoluer et enrichir leurs bases de connaissances en combinant de nouvelles connaissances aux connaissances existantes. Il apparaît ainsi qu'une combinaison de ressources internes, de réseaux externes et d'acquisitions de connaissances renforce la capacité des entreprises à créer de la valeur, de nouvelles connaissances et donc à innover. La capacité à créer de la valeur à long terme est jugée essentielle pour les entreprises et, plus particulièrement, les entreprises faisant de la R\&D dans les domaines d'activité intenses en connaissances.

Des travaux de recherche antérieurs ont proclamé que la combinaison d'approches à niveaux multiples, de tactiques complémentaires et d'apprentissage en synergie, contribue à renforcer l'ambidextrie organisationnelle (ANDRIOPOULOS et LEWIS 2009). De même, pour ANDERSON, COVIN et SLEVIN (2009), c'est la capacité des entreprises à être entrepreneuriales, en créant et en agissant sur les connaissances stratégiques, qui contribue à faire avancer l'apprentissage organisationnel, qui, au niveau de l'entreprise, s'illustre par l'encouragement des ingénieurs de tous métiers à concevoir et à prototyper leurs solutions innovatives. De plus, HOLMQVIST (2004) décrit les procédures relatives à l'apprentissage organisationnel comme une incitation à l'ouverture aux nouvelles idées, ce qui entraîne que les organisations entrent dans un mécanisme exploratoire, tel que l'expérimentation et le test, plutôt que rester dans l'utilisation routinière et répétée des connaissances existantes. COLOMBELLI, KRAFT et QUATRARO (2012) confirment ce point de vue, en déclarant que les entreprises à forte connaissance tendent à opérer en mode d'exploration de connaissances, tout en montrant des dispositions d'organisation d'activités de recherche plus souvent associées à un mode d'exploitation des connaissances. Les deux dynamiques de connaissances sont jugées candidates pour résoudre différents types de problèmes (à objectif simple ou multiple), avec l'implication de parties prenantes variées pour obtenir des résultats (CREPINSEK, LIU et MERNIK 2013). L'apprentissage bénéficie aux deux modes d'exploration ou d'exploitation des connaissances (SIREN, KOHTAMAKI et KUCKERTZ 2012). Selon ces auteurs, «le mécanisme d'apprentissage stratégique, à travers ses composantes intra-organisationnels qui rend possible la dissémination, l'interprétation et l'implantation de connaissances stratégiques, permet aux entreprises de capitaliser les bénéfices des deux modes d'exploration et d'exploitation ». MURRAY et O'MAHONY (2007) dénotent un manque de recherche dans ce domaine, mettant l'accent sur le besoin de comprendre comment les entreprises exploitent les opportunités et gèrent les défis posés par les connaissances collectées de part et 
d'autre des frontières organisationnelles . Essilor combine une innovation en mode « exploitation » sur les innovations incrémentales telles que Varilux ${ }^{\circledR}$ et Crizal ${ }^{\circledR}$ où le groupe a développé de solides processus de développement s'appuyant sur les compétences internes et une innovation en mode " exploration » sur des segments encore peu développés comme l'axe « Next generation consumers », pour lequel l'approche classique n'apporte pas toujours les réponses adaptées à la demande des 2.5 milliards de personnes encore non corrigées dans le monde.

\section{Gérer l'invention à travers l'innovation : construire un processus de gestion de la Propriété Intellectuelle robuste pour maintenir l'avantage concurrentiel}

La création de valeur dans le monde des affaires est conceptualisée de façon croissante à travers un prisme environnemental (HEARN et PACE 2006), qui témoigne d'une évolution dans notre compréhension et notre analyse des mécanismes d'innovation. D'un point de vue de chaîne de valeur, on analyse de plus en plus les relations et les mécanismes de création de valeur comme issus d'un écosystème, avec une focalisation sur les réseaux de valeur et sur la co-création de valeur (BEN LETAIFA, GRATACAP et ISCKIA 2013). Un argument de cette évolution de point de vue est que les approches de type écosystème mettent en valeur les interactions entre les acteurs, comme le confirment des études révélant le déplacement des interactions du marché des transactions vers les relations (GURAU 2009). En outre, la littérature sur le développement durable constitue le cadre pertinent pour analyser l'innovation et l'expansion du business, en montrant le rôle des nouvelles régulations qui poussent au développement des idées et des innovations amenant une croissance du business, ce qui constitue le modèle « regulatory push et vision pull » (PREUSS 2007). Dans cette perspective, les mécanismes de réitération et de boucle de réaction constituent un élément important des écologies de création de valeur, contribuant à instaurer des réseaux multidirectionnels comme inhérents à la création de valeur (HEARN et PACE 2006). Toutefois, la vue d'écosystème nécessite d'intégrer le risque de conflit qui peut émerger de tensions inter-entreprises (HEARN et PACE 2006), parce que l'écosystème de création de valeur suggère une portée beaucoup plus grande pour la capture et la création de valeur qui est construite de façon holistique plutôt qu'individuelle et qui va beaucoup plus loin qu'un partenariat d'entreprise à entreprise ou d'individu à individu visant à accroître les propres capacités de l'acteur sur le long terme. La formalisation et la contractualisation de clauses de brevets est un aspect clé des coopérations développées par Essilor, par exemple dans le domaine des équipements où, fréquemment, des concurrents partagent les mêmes fournisseurs de technologies spécifiques : ces dernières peuvent être, par exemple, des procédés de production innovants propriétaires développés par Essilor et de l'expertise technique apportée par le fournisseur, incluant les acquisitions. Dans ce contexte, les liens internes et les partenariats sont des éléments clés pour extraire le maximum de valeur de leurs innovations (ALCATER et ZHAO 2010). L'entreprise s'appuie notamment sur son écosystème dans le domaine des équipements, pour lesquelles les compétences en internes ne sont plus développées, travaillant avec ses fournisseurs de façon étroite, incluant le développement d'innovations par les fournisseurs.

La théorie du capital intellectuel suggère de plus qu'il existe des cas où les procédures et les technologies doivent être tenues à l'écart des partenaires en compétition (SOLITANDER et TIDSTROM 2010) pour éviter le risque de perte de l'avantage compétitif. ALCATER et ZHAO (2010) affirment que « tant que l'entreprise innovante peut intégrer les connaissances développées localement plus efficacement que leurs imitateurs potentiels, elles peuvent tirer bénéfice du faible coût dans les pays à faibles droits de propriété intellectuelle et donc continuer à réaliser un gain avec leurs innovations ». Pourtant, les entreprises n'ont pas toujours les moyens financiers pour investir dans de nouvelles formes de technologies, de systèmes et de connaissances, ce qui limite leurs possibilités de croissance. Par ailleurs, de telles configurations internationales nécessitent des formalités pour développer et soutenir des structures organisationnelles qui leur permettent de contrôler parfaitement leurs innovations, en s'engageant parfois dans les courses d'apprentissage ou de brevets. COLOMBELLI, KRAFT et QUATRARO (2012) rappellent que la création de technologie est souvent mandatée par une demande de brevet et qu'elle suit des cascades de résultats, 
apparaissant comme un moyen de financer des phases de création de connaissances. SALERNO et al. (2015) précisnt que, bien que les entreprises tendent à utiliser en interne une procédure linéaire d'innovation, les projets et les brevets associés venant d'un développement d'innovation impliquent habituellement différentes entreprises, chaque partie prenante ayant eu différents rôles, différentes responsabilités et différentes contributions. Les entreprises impliquées peuvent opérer sur des marchés différents, par exemple, servir des clients différents, utiliser les différentes technologies et bénéficier de conditions différentes de régulation, ce qui est le cas de Satisloh, un équipementier proposant des solutions de procédés et de machines pour l'industrie ophtalmique toute entière, acquise par Essilor en 2008 et servant déjà des concurrents.En plus d'influencer la nature, le spectre et l'extension de la collaboration entre les entreprises, de telles différences peuvent affecter les sorties de la procédure d'innovation, c'est-à-dire la protection par brevet et la commercialisation du produit innovant. Comme indiqué dans (MOSER 2013), existe-t-il un système optimal de droits de propriété intellectuelle propre à encourager l'innovation? La stratégie de propriété intellectuelle peut par exemple être partagée entre le développement d'innovations internes, et l'initiation d'innovation en externes avec les fournisseurs, dont la propriété est partagée au sein de l'écosystème de l'entreprise. En effet, les idées pour un nouveau business peuvent émerger en-deçà mais aussi au-delà des frontières de l'organisation. Les entreprises utilisent habituellement à la fois des sources internes et externes pour avoir accès aux informations, aux technologies, à l'innovation, aux pratiques du business et/ou au réseautage avec d'autres entreprises pour pouvoir accroitre croissance et profitabilité (NARAYANAN 2009). Dans de tels cas, les entreprises peuvent avoir besoin de nouveaux dispositifs, de nouveaux collaborateurs et de nouvelles connaissances (HUSSI 2004). De précédents travaux ont montré qu' "une stratégie d'innovation à succès nécessite un alignement précautionneux entre les sources de connaissances tant internes qu'externes » (ARORA, BELENZON et RIOS 2013), en suggérant que les connaissances techniques extensives d'une entreprise ne suffisent pas à combler les fossés de connaissances fonctionnelles. Ceci indique qu'une collaboration étroite, de nouvelles formes de partenariats et du réseautage de connaissances peuvent conduire à un apprentissage interorganisationnel et à la diffusion de nouvelles informations de valeur. POWELL et al. (2005) ont souligné l'importance de la participation à de tels réseaux à cause des facteurs clés de croissance qu'ils offrent, tels que l'accès aux nouvelles formes de connaissance, la fiabilité des informations et la capacité d'adaptation au changement. Cela affecte également les stratégies d'appropriation de connaissances (ALCATER et ZHAO 2010), où une firme peut rédiger de multiples demandes de brevets pour un produit donné (par exemple, les produits Varilux ${ }^{\circledR} S$ Series ${ }^{\circledR}$ sont protégés par une dizaine de brevets). La firme va notamment s'appuyer sur les connaissances internes en lien avec les innovations produits technologiques tout en recherchant également de nouveaux services créant de la valeur pour ses consommateurs afin de se différencier de ses concurrents.

Ainsi, et tandis que les ressources matérielles sont facilement accessibles ou imitées par les concurrents, les ressources immatérielles, telles que les connaissances, constituent un capital non évaluable conduisant l'entreprise à des réalisations intra-organisationnelles qui ne seront que plus tard transformées en succès commerciaux. De ce point de vue, les accords de confidentialité entre Essilor et ses partenaires de développement sont primordiaux pour garantir le succès de leurs relations quel que soit le degré de maturité du partenariat. HUSTED et VINTERGAARD (2004) ont attiré l'attention sur le potentiel offert par la transformation d'idées en inventions commercialement exploitables. La force des ressources incorporelles réside dans la coordination de ressources matérielles existantes pour engendrer des innovations et des avantages concurrentiels et donc sécuriser le développement continu de business. Les complexités d'appropriation de valeur introduites par l'engagement dans des activités technologiques collaboratives peut excéder leur potentiel d'augmentation de valeur (BELDERBOS et FAEMS 2010). Pour aller plus loin, il semble bien que le partage d'innovations que les entreprises choisissent de breveter varient selon les secteurs. En alternative à la protection par brevet, le secret industriel peut toujours être utilisé pour protéger des produits innovants ou des mécanismes d'amélioration de la production, tandis que les publications scientifiques peuvent couvrir la technologie innovante. 


\section{Conclusion}

$\mathrm{Au}$ cours de la dernière décennie, la littérature académique a régulièrement insisté sur le rôle des capacités dynamiques dans le maintien de la performance d'innovation des entreprises. Ce papier fournit de nombreux aperçus sur les méthodes et les défis de la gestion de l'innovation dans une entreprise internationale. Nous avons notamment décrit les différentes manières dont la société Essilor définit et soutient sa procédure d'innovation (en interne et/ou en externe), en mettant l'accent sur le rôle joué par l'environnement de travail interne dans le soutien au développement de l'innovation. Qui plus est, une vue de la connaissance de la dualité d'intérêts business dans la création et l'appropriation de la valeur est exprimée dans l'élaboration de la stratégie de propriété intellectuelle du groupe, en distinguant les flux de création de valeur (commercialisation de l'innovation) et d'appropriation de la valeur (brevet ou non de l'invention). L'article a également mentionné en quoi les procédures impliquant des partenaires externes diffèrent en termes d'étendue et de contraintes juridiques et en quoi elles ont des implications au-delà du mécanisme linéaire symbolisé par la séquence « de l'idée à l'innovation ». En particulier, en partageant des éléments de connaissances avec d'autres acteurs, l'entreprise Essilor vise et concourt à augmenter la création de valeur au niveau de son écosystème, tout en réduisant simultanément les coûts en partageant les ressources - et en bénéficiant de la capture d'objets de connaissance, notamment à travers les interactions avec ses fournisseurs. La connaissance apparaît ainsi comme un facteur clé dans l'identification des deux nouvelles sources de croissance interne et externe, ainsi que les possibilités d'innovation qu'elles représentent. Dans les réseaux d'entreprise, en particulier, il est suggéré que la connaissance est non seulement diffusée au sein du réseau, mais également combinée avec d'autres sources de connaissances (exclusive ou non exclusive) et construite sur la base d'un processus multidimensionnel et dynamique.

\section{Références bibliographiques}

AlCATER J., ZHAO M., Local R\&D strategies and multi-location firms: The role of internal linkages. Management Science, vol. 58, n 4, p. 734-753, 2010.

ANDERSON B. S., COVIN J.G., SLEVIN D.P., Understanding the relationship between entrepreneurial orientation and strategic learning capability: an empirical investigation. Strategic Entrepreneurship Journal, vol. 3, n 3, p. 218-240, 2009.

ANDRIOPOULOS C., LEWIS M.W., Exploitation-exploration tensions and organizational ambidexterity: Managing paradoxes of innovation. Organization Science, vol. 20, n 4, p. 696-717, 2009.

ARORA A., BELENZON S., Rios L.A., Make, buy, organize: The interplay between research, external knowledge, and firm structure. Strategic Management Journal, vol. 35, n 3, p. 317-337, 2013.

BELDERBos R., FAEMS D.L., Technological activities and their impact on the financial performance of the firm: Exploitation and exploration within and between firms. Journal of Product Innovation Management, vol. 27, $\mathrm{n}^{\circ}$ 6, p. 869-882, 2010.

Ben Letaifa S., GratACAP A., IsckiA T., Understanding Business Ecosystems: how firms succeed in the world of convergence. Bruxelles: De Boeck, 2013.

BRUNEel J., Yli-RENKO H., ClARYSSE B., Learning from experience and learning from others: how congenital and interorganizational learning substitute for experiential learning in young firm internationalization. Strategic entrepreneurship journal, vol. 4, n 2, p. 164-182, 2010.

BUTEL L., WATKINS A., Clusters of entrepreneurs: The application of ant colony optimisation modelling. Journal of Modelling in Management, vol. 1, n 3, p. 255-269, 2006.

COLOMBELli A., KRAFT J., QUATRARO F., High-growth firms and technological knowledge: do gazelles follow exploration or exploitation strategies? Industrial and Corporate Change, vol. 23, n 1, p. 261-291, 2012.

COOMS J.E., DEEDS D.L., DUANE IRELAND R., Placing the choice between exploration and exploitation in context: a study of geography and new product development. Strategic Entrepreneurship Journal, vol. 3, n 3, p. 261-279, 2009.

CREPINSEK M., LIU S.H., MERNIK M., Exploration and exploitation in evolutionary algorithms: A survey. ACM Computing Surveys (CSUR), vol. 45, $\mathrm{n}^{\circ}$ 3, p. 35, 2013.

DRUCKER P.F., Innovation and Entrepreneurship, 1985.

GURAU C., From Information Management to Knowledge Management. Encyclopedia of Information Science and Technology, 2009.

(c) 2016 ISTE OpenScience - Published by ISTE Ltd. London, UK - openscience.fr 
HARGADON A., SUTTON R.I., Technology brokering and innovation in a product development firm. Administrative science quarterly, p. 716-749, 1997.

HEARN G., PACE C., Value-creating ecologies: understanding next generation business systems. Foresight, vol. $8, \mathrm{n}^{\circ} 1$, p. 55-65, 2006.

HMIELSKI K.M., CORBETT A.C., The contrasting interaction effects of improvisational behavior with entrepreneurial selfefficacy on new venture performance and entrepreneur work satisfaction. ournal of business venturing, vol. $23, \mathrm{n}^{\circ} 4$, p. 482-496, 2008.

HOLMQVIST M., Experiential learning processes of exploitation and exploration within and between organizations: An empirical study of product development. Organization science, vol. 15, n 1, p. 70-81, 2004.

HUSSI T., Reconfiguring knowledge management-combining intellectual capital, intangible assets and knowledge creation. Journal of Knowledge Management, vol. 8, n 2, p. 36-52, 2004.

HUSTED K., VINTERGAARD C., Stimulating innovation through corporate venture bases. Journal of World Business, vol. 39, n 3, p. 296-306, 2004.

JOSHI K.D., CHI L., DATTA A., HAN S., Changing the competitive landscape: Continuous innovation through IT-enabled knowledge capabilities. Information Systems Research, vol. 21, n 3, p. 472-495, 2010.

KANE G.C., ALAVI M., Information technology and organizational learning: An investigation of exploration and exploitation processes. Organization Science, vol. 18, n 5, p. 796-812, 2007.

KETCHEN D.J., IRELAND R.D., SNOw C.C., Strategic entrepreneurship, collaborative innovation, and wealth creation. Strategic Entrepreneurship Journa, vol. 1, n 3-4, p. 371-385, 2007.

KURATKO D.F., HORNSBY J.S., COVIN J.G., Diagnosing a firm's internal environment for corporate entrepreneurship. Business Horizons, vol. 57, $\mathrm{n}^{\circ}$ 1, p. 37-47, 2013.

LAURSEN K., MASCARIELi F., PRENCIPE A., Regions matter: how localized social capital afects innovation and external knowledge acquisition. Organization Science, vol. 23, n 1, p. 177-193, $2012 \mathrm{~b}$.

MAURER I., BARTSCH V., EBERS M., The value of intra-organizational social capital: How it fosters knowledge transfer, innovation performance, and growth. Organization Studies, vol. 32, n 2, p. 157-185, 2011.

Moser P., Patents and innovation: Evidence from economic history. The Journal of Economic Perspectives, vol. 27, $\mathrm{n}^{\circ}$, p. 23-44, 2013.

MURRAY F., O’MAHONY S., Exploring the foundations of cumulative innovation: Implications for organization science. Organization Science, vol. 18, nº 6, p. 1006-1021, 2007.

NARAYANAN T.R., Academia-industry partnership: an impetus for strengthening teaching and research in higher education institutions. Current Science, vol. 96, nº 3, p. 343-346, 2009.

NICKERSON J.A., ZENGER T., A knowledge-based theory of the firm-The problem-solving perspective. Organization science, vol. $15, \mathrm{n}^{\circ} 6$, p. $617-632,2004$.

NONAKA I., TAKEUCHI H., The knowledge-creating company: How Japanese companies create the dynamics of innovation. Oxford University Press, 1995.

OBSTFELD D., Social networks, the tertius iungens orientation, and involvement in innovation. Administrative science quarterly, vol. 50, n 1, p. 100-130, 2005.

PETRINI M., POZZEBON M., Managing sustainability with the suport of business intelligence: Integrating socio-environmental indicators and organisational context. The Journal of Strategic Information, vol. 18, n 4, p. 178-191, 2009.

Powell W.W., White D.R., Koput K.W., OwEN-SMiTh J., Network dynamics and field evolution: The growth of interorganizational collaboration in the life sciences», American journal of sociology. vol. 110, n 4, p. 1132-1205, 2005.

PREUSS L., Contribution of purchasing and supply management to ecological innovation. International Journal of Innovation Management, vol. 11, n 4, p. 515-537, 2007.

PROVOST F., FAWCETT T., Data Science for Business: What you need to know about data mining and data-analytic thinking. O’Reilly Media Inc, 2013.

Quintane E., Mitch Casselman R., Sebastian Reiche B., Nylund P.A., Innovation as a knowledge-based outcome. Journal of Knowledge Management, vol. 15, nº 6, p. 928-947, 2011.

SAINT-ONGE H., Tacit knowledge the key to the strategic alignment of intellectual capital. Planning Review, vol. $24, \mathrm{n}^{\circ} 2$, p. 10-16, 1996. 
SALERNO M.S., VASCONCEloS GOMES L.A., SilvA D.O., BAGNO R.B., FREITAS S.L., Innovation processes: Which process for which project. Technovation, vol. 35, p. 59-70, 2015.

SCHULz M., Pathways of relevance: Exploring inflows of knowledge into subunits of multinational corporations. Organization Science, vol. 14, nº 4, p. 440-459, 2003.

SHANE S.A., A general theory of entrepreneurship: The individual-opportunity nexus. Edward Elgar Publishing, 2003.

SiREN C.A., KOHTAMAKI M., KUCKERTZ A., Exploration and exploitation strategies, profit performance, and the mediating role of strategic learning: Escaping the exploitation trap. Strategic Entrepreneurship Journal, vol. 6, nº 1, p. 18-41, 2012.

SOlitAnder M., TidSTROM A., Competitive flows of intellectual capital in value creating networks. Journal of intellectual capital, vol. 11, n 1, p. 23-38, 2010.

TidD J., Bessant J., PAVITT K., Management de l'innovation ; intégration du changement technologique, commercial et organisationnel. Bruxelles : De Boeck, 2006.

VAsudeva G., SPEnCER J., TeEgen H., Bringing the institutional context back in: A cross-national comparison of alliance partner selection and knowledge acquisition. Organization Science, vol. 24, n 2, p. 319-338, 2013. 